\title{
WEIGHTED INEQUALITIES FOR MAXIMAL FUNCTIONS ASSOCIATED WITH GENERAL MEASURES
}

\author{
KENNETH F. ANDERSEN
}

\begin{abstract}
For certain positive Borel measures $\mu$ on $\mathbf{R}$ and for $T_{\mu}$ any of three naturally associated maximal function operators of Hardy-Littlewood type, the weight pairs $(u, v)$ for which $T_{\mu}$ is of weak type $(p, p), 1 \leq p<\infty$, and of strong type $(p, p), 1<p<\infty$, are characterized. Only minimal assumptions are placed on $\mu$; in particular, $\mu$ need not satisfy a doubling condition nor need it be continuous.
\end{abstract}

\section{INTRODUCTION}

Let $\mu$ be a positive Borel measure on $\mathbf{R}$ which is finite on bounded sets. Note that $\mu$ is regular [10, p. 208]. The maximal function operator $M_{\mu}$ is defined for locally integrable $f$ and $x \in \mathbf{R}$ by

$$
M_{\mu} f(x)=\sup \frac{1}{\mu(I)} \int_{I}|f| d \mu,
$$

the supremum being taken over all intervals $I$ containing $x$. The one-sided maximal function operators $M_{\mu}^{+}, M_{\mu}^{-}$are defined similarly except that the supremum is taken over intervals of the form $[x, x+h)$ and $(x-h, x]$, $h>0$, respectively. Quotients of the form $0 / 0$ are taken to be zero.

If $T$ is any one of these operators and $1 \leq p<\infty$, then the weak type inequality

$$
\int_{\{x: T f(x)>\lambda\}} u d \mu \leq C \lambda^{-p} \int|f|^{p} v d \mu \quad \forall f \in L^{p}(v d \mu)
$$

and the strong type inequality

$$
\int|T f|^{p} u d \mu \leq C \int|f|^{p} v d \mu \quad \forall f \in L^{p}(v d \mu)
$$

for $1<p<\infty$ are known to hold for $u=v=1$ (see [9, 2]). The purpose of this paper is to characterize those pairs $(u, v)$ of nonnegative weight functions for which (1.1) and (1.2) hold.

Received by the editors September 12, 1989 and, in revised form, February 14, 1990. The results of this paper were presented to the Special Session on Singular Integral Operators and Related Areas held at the 856th meeting of the American Mathematical Society, Fayetteville, Arkansas, March 23-24, 1990.

1980 Mathematics Subject Classification (1985 Revision). Primary 42B25.

Key words and phrases. Maximal functions, weighted inequalities, weak type, strong type.

Research supported in part by NSERC grant \#A-8185. 
Inequalities of this type are intimately connected with the differentiation of integrals and are also of interest because of their application in obtaining boundedness properties of other operators commonly studied in analysis. Although (1.1) and (1.2) have been widely studied, the weight pairs have been characterized only for measures satisfying considerably stronger hypotheses than those assumed here.

If $\mu\{x\}=0$ for all $x \in \mathbf{R}$ and $T=M_{\mu}$, B. Muckenhoupt [5] characterized the two weight function pairs $(u, v)$ for which (1.1) holds and the single weight functions $u$ for which $(u, u)$ satisfies (1.2).

E. Sawyer [6] characterized the two weight function pairs $(u, v)$ satisfying (1.2) for $M_{\mu}$ provided $\mu$ satisfies a doubling condition.

For $M_{\mu}^{+}$and $M_{\mu}^{-}$, Sawyer [8] characterized (1.2) in the case $\mu$ is Lesbesgue measure, while F. J. Martín-Reyes, P. Ortega Salvador, and A. de la Torre [4] treated (1.1) and (1.2) for $\mu$ absolutely continuous with respect to Lebesgue measure with (strictly) positive derivative.

Theorem 1 characterizes the weak type inequality (1.1).

Theorem 1. Let $\mu$ be a positive Borel measure which is finite on bounded sets, $1 \leq p<\infty$, and $u, v$ nonnegative weight functions on $\mathbf{R}$. Then

(a) $T=M_{\mu}^{+}$satisfies (1.1) if and only if $(u, v) \in A_{p}^{+}(\mu)$, that is, there is a constant $K$ such that

$$
\left(\int_{(a, b]} u d \mu\right)^{1 / p}\left(\int_{[b, c)} v^{-1 /(p-1)} d \mu\right)^{1 / p^{\prime}} \leq K \mu(a, c)
$$

for all $-\infty<a<b<c<\infty$.

(b) $T=M_{\mu}^{-}$satisfies (1.1) if and only if $(u, v) \in A_{p}^{-}(\mu)$, that is, there is a constant $K$ such that

$$
\left(\int_{[b, c)} u d \mu\right)^{1 / p}\left(\int_{(a, b]} v^{-1 /(p-1)} d \mu\right)^{1 / p^{\prime}} \leq K \mu(a, c)
$$

for all $-\infty<a<b<c<\infty$.

(c) $T=M_{\mu}$ satisfies (1.1) if and only if $(u, v) \in A_{p}(\mu)$, that is, there is a constant $K$ such that

$$
\left(\int_{(a, b)} u d \mu\right)^{1 / p}\left(\int_{(a, b)} v^{-1 /(p-1)} d \mu\right)^{1 / p^{\prime}} \leq K \mu(a, b)
$$

for all $-\infty<a<b<\infty$.

In each of (1.3), (1.4), (1.5), products of the form $0 \cdot \infty$ are taken to be zero, while for $p=1$ expressions of the form $\left(\int_{I} v^{-1 /(p-1)} d \mu\right)^{1 / p^{\prime}}$ are interpreted as $\mu$-ess. $\sup _{x \in I} 1 / v(x)$.

Examples of weights satisfying $A_{p}(\mu), A_{p}^{+}(\mu), A_{p}^{-}(\mu)$ are easily constructed. Note in particular that $(u, u) \in A_{p}^{+}(\mu)$ if $u$ is nondecreasing, while $(u, u) \in$ $A_{p}^{-}(\mu)$ if $u$ is nonincreasing. Further, $A_{p}(\mu)=A_{p}^{+}(\mu) \cap A_{p}^{-}(\mu)$.

Theorem 2 characterizes the strong type inequalities (1.2). 
Theorem 2. Let $\mu$ be a positive Borel measure which is finite on bounded sets, $1<p<\infty$, and $u, v$ nonnegative weight functions on $\mathbf{R}$. Then

(a) $T=M_{\mu}^{+}$satisfies (1.2) if and only if $(u, v) \in S_{p}^{+}(\mu)$, that is, there is a constant $K$ such that for every $-\infty<a<b<\infty$ with $\int_{(-\infty, a]} u d \mu>0$

$$
\int_{[a, b)}\left|M_{\mu}^{+} \chi_{[a, b)} v^{-1 /(p-1)}\right|^{p} u d \mu \leq K \int_{[a, b)} v^{-1 /(p-1)} d \mu<\infty .
$$

(b) $T=M_{\mu}^{-}$satisfies (1.2) if and only if $(u, v) \in S_{p}^{-}(\mu)$, that is, there is a constant $K$ such that for every $-\infty<a<b<\infty$ with $\int_{[b, \infty)} u d \mu>0$

$$
\int_{(a, b]}\left|M_{\mu}^{-} \chi_{(a, b]} v^{-1 /(p-1)}\right|^{p} u d \mu \leq K \int_{(a, b]} v^{-1 /(p-1)} d \mu<\infty .
$$

(c) $T=M_{\mu}$ satisfies (1.2) if and only if $(u, v) \in S_{p}(\mu)$, that is, there is a constant $K$ such that for every $-\infty<a<b<\infty$

$$
\int_{(a, b)}\left|M_{\mu} \chi_{(a, b)} v^{-1 /(p-1)}\right|^{p} u d \mu \leq K \int_{(a, b)} v^{-1 /(p-1)} d \mu<\infty .
$$

For the single weight function case, $u=v$, and $p>1$, the inequalities (1.1) and (1.2) are equivalent as the following theorem shows.

Theorem 3. Let $\mu$ be a positive Borel measure which is finite on bounded sets, $1<p<\infty$, and $u$ a nonnegative weight function on $\mathbf{R}$. Then

(a) $(u, u) \in A_{p}^{+}(\mu)$ if and only if $(u, u) \in S_{p}^{+}(\mu)$.

(b) $(u, u) \in A_{p}^{-}(\mu)$ if and only if $(u, u) \in S_{p}^{-}(\mu)$.

(c) $(u, u) \in A_{p}(\mu)$ if and only if $(u, u) \in S_{p}(\mu)$.

The proof of Theorem 1 depends on the following lemma, of independent interest, which generalizes a weak type inequality for operators of Hardy type considered in $[1,7]$.

Lemma. Let $\mu$ be a positive Borel measure on $\mathbf{R}$ which is finite on bounded sets and let $1<p<\infty$.

(a) Suppose $\mu[0, x]>0$ for all $x \geq 0$ and

$$
P_{\mu} f(x)=\frac{1}{\mu[0, x]} \int_{[0, x]} f d \mu, \quad x \geq 0 .
$$

There is a constant $A$ such that for all $f \in L^{p}(v d \mu)$

$$
\int_{\left\{x \geq 0:\left|P_{\mu} f(x)\right|>\lambda\right\}} u d \mu \leq A \lambda^{-p} \int_{[0, \infty)}|f|^{p} v d \mu
$$

if and only if there is a constant $K$ such that

$$
\left(\int_{[a, b)} u d \mu\right)^{1 / p}\left(\int_{[0, a]} v^{-1 /(p-1)} d \mu\right)^{1 / p^{\prime}} \leq K \mu[0, b)
$$

for all $0 \leq a<b<\infty$. Moreover, the smallest constants $A$ in (1.9) and $K$ in (1.10) satisfy $C A \leq K^{p} \leq A$ for a constant $C$ depending only on $p$. 
(b) Suppose $\mu(0, x]>0$ for all $x>0$ and

$$
P_{\mu} f(x)=\frac{1}{\mu(0, x]} \int_{(0, x]} f d \mu, \quad x>0 .
$$

There is a constant $A$ such that for all $f \in L^{p}(v d \mu)$

$$
\int_{\left\{x>0:\left|P_{\mu} f(x)\right|>\lambda\right\}} u d \mu \leq A \lambda^{-p} \int_{(0, \infty)}|f|^{p} v d \mu
$$

if and only if there is a constant $K$ such that

$$
\left(\int_{[a, b)} u d \mu\right)^{1 / p}\left(\int_{(0, a]} v^{-1 /(p-1)} d \mu\right)^{1 / p^{\prime}} \leq K \mu(0, b)
$$

for all $0<a<b<\infty$. Moreover, the smallest constants $A$ in (1.11) and $K$ in (1.12) satisfy $C A \leq K^{p} \leq A$ for a constant $C$ depending only on $p$.

The Lemma is proved in $\S 2$ and the proofs of the theorems follow in $\S \S 3-$ 5. The letter $C$ will be used to denote constants, possibly depending on $p$, which may not be the same from line to line. For convenience of notation, when $p>1$, set $\sigma=v^{-1 /(p-1)}$. The length of an interval $J$ is denoted $|J|$. An interval $J$ with endpoints $a<b$ which is open at $a$ will be denoted by $(a, b\}$; thus $(a, b\}=(a, b)$ if $J=(a, b)$ and $(a, b\}=(a, b]$ if $J=\{a, b]$.

\section{Proof of the Lemma}

We shall give the proof only for (a) since the proof for $(b)$ is entirely similar.

Suppose first that (1.9) holds and fix $0 \leq a<b<\infty$. If $I \equiv \int_{[0, a]} \sigma d \mu=\infty$, Hölder's inequality shows there is $g \geq 0$ supported on $[0, a]$ with $\int g^{p} v d \mu<$ $\infty$ but $\int g d \mu=\infty$. Thus $P_{\mu} g(x)=\infty$ for $x \geq a$, so (1.9) forces $\int_{[a, \infty)} u d \mu$ $=0$ and (1.10) holds by convention. If $0<I<\infty$, let $f=\chi_{[0, a]} \sigma$. Then for $a \leq x<b, P_{\mu} f(x) \geq(\mu[0, b))^{-1} \int_{[0, a]} \sigma d \mu=(\mu[0, b))^{-1} I$, so for all $B<1$ (1.9) shows

$$
\int_{[a, b)} u d \mu \leq A(\mu[0, b))^{p}(B I)^{-p} \int_{[0, a]} f^{p} v d \mu=A(\mu[0, b))^{p} B^{-p} I^{1-p}
$$

and (1.10) follows since $B<1$ is arbitrary. Thus (1.9) implies (1.10).

Suppose now that (1.10) holds. The Lorentz space $L^{p, q}(u d \mu), 1<p<\infty$, $1 \leq q \leq \infty$, consists of those $f$ for which the quasi-norm $\|f\|_{p, q}<\infty$, where

$$
\|f\|_{p, q}= \begin{cases}\left(\int_{0}^{\infty} \frac{q}{p} t^{q / p-1} f^{*}(t)^{q} d t\right)^{1 / q}, & q<\infty, \\ \sup _{\lambda>0} \lambda\left(\int_{\{x \geq 0:|f(x)|>\lambda\}} u d \mu\right)^{1 / p}, & q=\infty,\end{cases}
$$

and $f^{*}$ denotes the nonincreasing, equimeasurable rearrangement of $f$ onto $(0, \infty)$. Thus,

$$
\left\|\frac{\chi_{[a, \infty)}(\cdot)}{\mu[0, \cdot]}\right\|_{p, \infty}=\sup _{b>a} \frac{1}{\mu[0, b)}\left(\int_{[a, b)} u d \mu\right)^{1 / p}
$$


so (1.10) becomes

$$
\left\|\frac{\chi_{[a, \infty)}(\cdot)}{\mu[0, \cdot]}\right\|_{p, \infty}\left(\int_{[0, a]} \sigma d \mu\right)^{1 / p^{\prime}} \leq K .
$$

As in [7, Proof of Theorem 4], a duality argument shows that (1.9) is equivalent to

$$
\int_{[0, \infty)} G^{p^{\prime}} \sigma d \mu \leq C A\|g\|_{p^{\prime}, 1}^{p^{\prime}}
$$

where

$$
G(x)=\int_{[x, \infty)} \frac{g(t)}{\mu[0, t]} u(t) d \mu(t)
$$

for $g \geq 0, g \in L^{p^{\prime}, 1}(u d \mu)$.

Now

$$
G(x) \leq C\left\|\frac{\chi_{[x, \infty)}(\cdot)}{\mu[0, \cdot]}\right\|_{p, \infty}\|g\|_{p^{\prime}, 1},
$$

so (2.1) shows that $G(x)<\infty$ whenever $\int_{[0, x]} \sigma d \mu>0$. Since (2.2) is trivial if $\int_{[0, x]} \sigma d \mu=0$ for all $x$, we may assume that $x_{0}=\inf \left\{x \geq 0: \int_{[0, x]} \sigma d \mu>\right.$ $0\}<\infty$. Then $G\left(x_{0}\right)<\infty$ unless $\int_{\left[0, x_{0}\right]} \sigma d \mu=0$ and $G(x)$ is finite and nonincreasing on $\left(x_{0}, \infty\right)$ with $\lim _{x \rightarrow \infty} G(x)=0$. Hence (2.3) and (2.1) show

$$
\int_{\left[0, x_{0}\right]} G^{p^{\prime}} \sigma d \mu=G\left(x_{0}\right)^{p^{\prime}} \int_{\left[0, x_{0}\right]} \sigma d \mu \leq C K^{p^{\prime}}\|g\|_{p^{\prime}, 1}^{p^{\prime}}
$$

and it suffices to prove

$$
\int_{\left(x_{0}, \infty\right)} G^{p^{\prime}} \sigma d \mu \leq C K^{p^{\prime}}\|g\|_{p^{\prime}, 1}^{p^{\prime}}
$$

For each integer $j$, set $a_{j}=\inf \left\{x \geq x_{0}: G(x) \leq 2^{j}\right\}$. Then $G(x) \leq 2^{j}$ for $x \in\left(a_{j}, \infty\right)$ and

$$
\int_{\left[a_{j}, a_{j-1}\right]} \frac{g(t)}{\mu[0, t]} u(t) d \mu(t) \geq 2^{j-1} \text { if } a_{j}>x_{0} .
$$

Let $J=\left\{j: a_{j}<a_{j-1}\right\}$ and set $\chi_{j}=\chi_{\left[a_{j-1}, a_{j-2}\right]}$. Then

$$
\begin{aligned}
\int_{\left(x_{0}, \infty\right)} G^{p^{\prime}} \sigma d \mu & =\sum_{j \in J} \int_{\left(a_{j}, a_{j-1}\right]} G^{p^{\prime}} \sigma d \mu \leq \sum_{j \in J} 2^{j p^{\prime}} \int_{\left[0, a_{j-1}\right]} \sigma d \mu \\
& \leq 4^{p^{\prime}} \sum_{j \in J}\left(\int_{[0, \infty)} \chi_{j}(t) \frac{g(t)}{\mu[0, t]} u(t) d \mu(t)\right)^{p^{\prime}}\left(\int_{\left[0, a_{j-1}\right]} \sigma d \mu\right) \\
& \leq C \sum_{j \in J}\left\|\chi_{j} g\right\|_{p^{\prime}, 1}^{p^{\prime}}\left\|\frac{\chi_{\left[a_{j-1}, \infty\right)}(\cdot)}{\mu[0, \cdot]}\right\|_{p, \infty}^{p^{\prime}}\left(\int_{\left[0, a_{j-1}\right]} \sigma d \mu\right) \\
& \leq C K^{p^{\prime}} \sum_{j \in J}\left\|\chi_{j} g\right\|_{p^{\prime}, 1}^{p^{\prime}}
\end{aligned}
$$


by (2.1). Thus

$$
\int_{\left(x_{0}, \infty\right)} G^{p^{\prime}} \sigma d \mu \leq C K^{p^{\prime}}\|g\|_{p^{\prime}, 1}^{p^{\prime}}
$$

by [3, Lemma 2.5] since $\sum_{j \in J} \chi_{j}(x) \leq 2$. This proves (2.4) and completes the proof of the Lemma.

\section{Proof of Theorem 1}

Suppose (1.1) holds for $M_{\mu}^{+}$, and let $-\infty<a<b<c<\infty$ and $I=$ $\left(\int_{[b, c)} v^{-1 /(p-1)} d \mu\right)^{1 / p^{\prime}}$. If $I=0,(1.3)$ holds by convention. If $I=\infty$, there is $g \geq 0$ supported on $[b, c)$ with $\int g^{p} v d \mu<\infty$ but $\int g d \mu=\infty$. Then $M_{\mu}^{+} g(x)=\infty$ for all $x \leq b$ so (1.1) forces $\int_{(a, b]} u d \mu=0$ and (1.3) holds in this case also. Suppose $0<I<\infty$. If $p>1$, let $f=\sigma \chi_{[b, c)}$ so that $M_{\mu}^{+} f(x) \geq I(\mu(a, c))^{-1}$ for $x \in(a, b]$. Thus (1.1) yields $\int_{(a, b]} u d \mu \leq$ $C I^{1-p}(\mu(a, c))^{p}$ which is (1.3). If $p=1$, let $\varepsilon>0$ and select $E \subset[b, c)$ with $\mu(E)>0$ such that $v(t)<\varepsilon+I$ for $t \in E$. Then $M_{\mu}^{+} \chi_{E}(x) \geq \mu(E)(\mu(a, c))^{-1}$ for $x \in(a, b]$ so $(1.1)$ yields $\int_{(a, b]} u d \mu \leq C(\varepsilon+I) \mu(a, c)$. Since $\varepsilon>0$ was arbitrary, this yields (1.3). This proves the necessity of (1.3) for (1.1) with $T=M_{\mu}^{+}$.

To prove (1.1) for $T=M_{\mu}^{+}$, it suffices to consider $f \in L^{p}(v d \mu), f \geq 0$ and of bounded support. Fix an integer $N$ and for $\lambda>0$ set $\Omega=\Omega_{\lambda, N}=$ $\left\{x>N: M_{\mu}^{+} f(x)>\lambda\right\}$. Since $M_{\mu}^{+} f(x)$ is left lower semicontinuous and $\Omega$ is bounded, the component intervals $I$ of $\Omega$ are of the form $(a, b)$ or $(a, b]$ for $-\infty<a<b<\infty$.

Let $I=(a, b]$ be a component interval of $\Omega$. Then there is $b_{j} \searrow b$ with $b_{j} \notin \Omega$ so for all $c>b, \int_{\left[b_{j}, c\right)} f d \mu \leq \lambda \mu\left[b_{j}, c\right)$ and hence also

$$
\int_{(b, c)} f d \mu \leq \lambda \mu(b, c) .
$$

On the other hand, for $x \in(a, b]$ there is $c>x$ such that

$$
\int_{[x, c)} f d \mu>\lambda \mu[x, c)
$$

We may assume $c>b$, for otherwise if

$$
c_{0}=\sup \left\{c>x: \int_{[x, c)} f d \mu>\lambda \mu[x, c)\right\} \leq b,
$$

we have $c_{0} \in \Omega$ so there is $c_{1}>c_{0}$ with $\int_{\left[c_{0}, c_{1}\right)} f d \mu>\lambda \mu\left[c_{0}, c_{1}\right)$ and hence $\int_{\left[x, c_{1}\right)} f d \mu>\lambda\left[x, c_{1}\right)$, which contradicts the definition of $c_{0}$. Subtracting (3.1) from (3.2) then yields

$$
\int_{[x, b\}} f d \mu>\lambda \mu[x, b\}>0 \quad \forall x \in(a, b\}
$$


for the case $(a, b\}=(a, b]$; a similar argument shows $(3.3)$ for $(a, b\}=$ $(a, b)$.

Suppose now that (1.3) holds and $p>1$. Observe first that the Dominated Convergence Theorem shows that $(1.3)$ also holds if the intervals $[b, c)$ and $(a, c)$ are replaced by $[b, c]$ and $(a, c]$ respectively. Thus

$$
\left(\int_{(a, b]} u d \mu\right)^{1 / p}\left(\int_{[b, c\}} \sigma d \mu\right)^{1 / p^{\prime}} \leq K \mu(a, c\}
$$

for all $-\infty<a<b<c<\infty$.

Now (3.3) shows

$$
I \subset F=\left\{x \in(-\infty, b\}: \frac{1}{\mu[x, b\}} \int_{[x, b]} \chi_{I} f d \mu>\lambda\right\} .
$$

A linear change of variable in the Lemma then shows

$$
\int_{I} u d \mu \leq \int_{F} u d \mu \leq C K_{1}^{p} \lambda^{-p} \int_{(-\infty, b\}} \chi_{I} f^{p} v d \mu
$$

where

$$
K_{1}=\sup \frac{1}{\mu(d, b\}}\left(\int_{(d, c]} u d \mu\right)^{1 / p}\left(\int_{[c, b\}} \sigma d \mu\right)^{1 / p^{\prime}},
$$

the supremum being taken over $d<c \leq b$ if $(a, b\}=(a, b]$ and over $d<$ $c<b$ if $(a, b\}=(a, b)$. In any case, from (3.4), $K_{1} \leq K$ and hence

$$
\int_{I} u d \mu \leq C K^{p} \lambda^{-p} \int_{I} f^{p} v d \mu
$$

Summing over all component intervals $I$ and letting $N \rightarrow-\infty$ then yields (1.1). This completes the proof of (1.1) for $p>1$.

Suppose now that $p=1$ and (1.3) holds. Observe first that (1.3) is equivalent to $M_{\mu}^{-} u(x) \leq K v(x)$ for $\mu$-almost all $x$.

For $x \in(a, b\},(3.3)$ shows that

$$
\left(\mu \text {-ess. } \sup _{[x, b\}} v^{-1}\right) \int_{[x, b\}} f v d \mu \geq \lambda \mu[x, b\}>0,
$$

so $\mu$-ess. $\sup _{[x, b\}} v^{-1}>0$. Then (1.3) implies $\int_{[x, b\}} u d \mu<\infty$ and $M_{\mu}^{-} u(x)<$ $\infty$ for $\mu$-almost all $x \in(a, b)$. The weight

$$
w(x)=\mu \text {-ess. } \inf _{y \in[x, b\}}\left(M_{\mu}^{-} \chi_{(a, b\}} u\right)(y)
$$

is nondecreasing on $(a, b\}$ and $w(x) \leq K v(x)$ for $\mu$-almost all $x \in(a, b\}$. 
For $a<a_{1}<b$, Fubini's Theorem and (3.3) show

$$
\begin{aligned}
\int_{\left[a_{1}, b\right\}} f w d \mu & =\int_{\left[a_{1}, b\right\}} f(x)\left[w\left(a_{1}\right)+\int_{\left[a_{1}, x\right]} d w(t)\right] d \mu(x) \\
& =w\left(a_{1}\right) \int_{\left[a_{1}, b\right\}} f d \mu+\int_{\left[a_{1}, b\right\}} d w(t) \int_{[t, b\}} f(x) d \mu(x) \\
& \geq w\left(a_{1}\right) \lambda \int_{\left[a_{1}, b\right\}} d \mu+\int_{\left[a_{1}, b\right\}} d w(t) \lambda \int_{[t, b\}} d \mu(x) \\
& =\lambda \int_{\left[a_{1}, b\right\}} w d \mu .
\end{aligned}
$$

Letting $a_{1} \searrow a$ yields

$$
\int_{(a, b\}} f w d \mu \geq \lambda \int_{(a, b\}} w d \mu .
$$

It will suffice to prove

$$
\int_{(a, b\}} w d \mu \geq \int_{(a, b\}} u d \mu
$$

for then

$$
\int_{(a, b\}} u d \mu \leq \int_{(a, b\}} w d \mu \leq \frac{1}{\lambda} \int_{(a, b\}} f w d \mu \leq \frac{K}{\lambda} \int_{(a, b\}} f v d \mu,
$$

so that upon summing over all component intervals $(a, b\}$ and letting $N \rightarrow$ $-\infty,(1.1)$ is obtained.

To prove (3.5), we first show that for fixed $B<1$,

$$
\begin{aligned}
& \text { if } x \in(a, b\} \text { with } w(x)>0 \text {, either } \mu\{x\}>0 \text { with } B u(x)<w(x) \\
& \text { or } \exists y \in(x, b\} \text { with } B \int_{[x, y]} u d \mu \leq w(x) \mu[x, y] \leq \int_{[x, y]} w d \mu .
\end{aligned}
$$

To see this, select $E \subset[x, b]$ with $\mu(E)>0$ such that $y \in E$ implies $\left(M_{\mu}^{-} \chi_{(a, b\}} u\right)(y)<w(x) / B$. Then

$$
(\mu(c, y])^{-1} \int_{(c, y]} \chi_{(a, b\}} u d \mu \leq\left(M_{\mu}^{-} \chi_{(a, b\}} u\right)(y)
$$

for $c<y$, so upon letting $c \nearrow x$, it follows that either $\mu[x, y]=0$ or $B \int_{[x, y]} u d \mu<w(x) \mu[x, y]$ and this implies (3.6).

Now fix $z \in(a, b\}$ with $w(z)>0$. Then (3.6) shows that $S=\{y \in[z, b\}$ : $\left.\int_{[z, y]} w d \mu \geq B \int_{[z, y]} u d \mu\right\}$ contains $z$. If $y_{0}=\sup S$, then

$$
\int_{\left[z, y_{0}\right)} w d \mu \geq B \int_{\left[z, y_{0}\right)} u d \mu .
$$

We shall show that $y_{0}=b$. If $y_{0}<b$, the definition of $y_{0}$ together with (3.6) shows that we must have $\mu\left\{y_{0}\right\}>0$ with $B u\left(y_{0}\right)<w\left(y_{0}\right)$. But then (3.7) yields $\int_{\left[z, y_{0}\right]} w d \mu>B \int_{\left[z, y_{0}\right]} u d \mu$ and the Dominated Convergence Theorem 
then shows $y_{0}+\varepsilon \in S$ for small $\varepsilon>0$. This contradicts the definition of $y_{0}$. Hence $y_{0}=b$. Moreover, we now have

$$
\int_{[z, b\}} w d \mu \geq B \int_{[z, b\}} u d \mu
$$

for if $(a, b\}=(a, b)$ this is just (3.7) and if $(a, b\}=(a, b]$ then $\mu\{b\}>0$, so (3.6) and (3.7) yield (3.8).

If $w(z)=0$ for some $z \in(a, b\}$, let $z_{0}=\sup \{z: w(z)=0\}$. Then $\int_{\left(a, z_{0}\right)} w d \mu=\int_{\left(a, z_{0}\right)} u d \mu=0$, and if $u\left(z_{0}\right) \mu\left\{z_{0}\right\}>0$, then $w\left(z_{0}\right)>0$ also since $\left(M_{\mu}^{-} \chi_{(a, b\}} u\right)(y) \geq u\left(z_{0}\right) \mu\left\{z_{0}\right\} / \mu(a, b\}>0$ for all $y \in\left[z_{0}, b\right\}$. This shows that (3.8) holds for all $z \in(a, b\}$. Since $B<1$ was arbitrary, this proves (3.5) and completes the proof of (1.1) for $p=1$.

The proof of part (a) is complete. The proof of part (b) is similar and is therefore omitted. For part (c), the proof that (1.1) for $M_{\mu}$ implies (1.5) is similar to the proof which showed (1.1) for $M_{\mu}^{+}$implies (1.3). If (1.5) holds, then both (1.3) and (1.4) hold, so (1.1) for $M_{\mu}$ follows from parts (a) and (b) since $M_{\mu} f \leq M_{\mu}^{+} f+M_{\mu}^{-} f$.

\section{Proof of Theorem 2}

Suppose $T=M_{\mu}^{+}$satisfies (1.2) and $-\infty<a<b<\infty$ with $\int_{(-\infty, a]} u d \mu>$ 0 . If $\int_{[a, b)} \sigma d \mu=\infty$, there is $g \geq 0$ supported on $[a, b)$ with $\int g^{p} v d \mu<$ $\infty$ but $\int g d \mu=\infty$. Then $M_{\mu}^{+} g(x)=\infty$ for all $x \leq a$ so (1.2) forces $\int_{(-\infty, a]} u d \mu=0$, a contradiction. Thus $\int_{[a, b)} \sigma d \mu<\infty$ and upon selecting $f=\sigma \chi_{[a, b)}$ in (1.2) we obtain (1.6), i.e., $(u, v) \in S_{p}^{+}(\mu)$.

Suppose now that $(u, v) \in S_{p}^{+}(\mu)$ and $\varepsilon>0$. If $\int_{(-\infty, x]} u d \mu>0$ for all $x$, let $I_{\varepsilon}=(-\infty, \infty)$; otherwise, let $x_{0}=\sup \left\{x: \int_{(-\infty, x]} u d \mu=0\right\}$ and set $I_{\varepsilon}=\left[x_{0}+\varepsilon, \infty\right)$ or $\left[x_{0}, \infty\right)$ according as $\int_{\left(-\infty, x_{0}\right]} u d \mu$ is zero or positive. Then for $-\infty<a<b<\infty$, with $a \in I_{\varepsilon}$, (1.6) shows that

$$
\int_{[a, b)} \sigma d \mu<\infty
$$

and simple convergence arguments show that (1.6) still holds with the same constant $K$ if $[a, b)$ is replaced throughout by any interval $J$ of finite length with left endpoint in $I_{\varepsilon}$.

To prove (1.2) for $M_{\mu}^{+}$it suffices to consider $f \in L^{p}(v d \mu)$ with $f \geq 0$, $f$ bounded and with bounded support. For such $f$ and fixed $N$, the sets $\Omega_{k}=\left\{x>N: M_{\mu}^{+} f(x)>2^{k}\right\}$ are uniformly bounded for $k \in \mathbf{Z}$. The component intervals $I_{j, k}=\left(a_{j, k}, b_{j, k}\right\}$ of $\Omega_{k}$ satisfy

$$
\int_{\left[x, b_{j, k}\right\}} f d \mu>2^{k} \mu\left[x, b_{j, k}\right\}>0 \quad \forall x \in I_{j, k} .
$$


Let $E_{k}=\Omega_{k} \backslash \Omega_{k+1}, E_{j, k}=I_{j, k} \backslash \Omega_{k+1}$, and $F_{j, k}=I_{\varepsilon} \cap E_{j, k}$. Then Hölder's inequality applied to (4.2) shows that $\int_{\left[x, b_{j, k}\right\}} \sigma d \mu>0$ for $x \in I_{j, k}$. This combined with (4.1) gives

$$
0<\int_{\left[x, b_{j, k}\right\}} \sigma d \mu<\infty \quad \forall x \in F_{j, k}
$$

and hence (4.2) shows

$$
\begin{gathered}
\int_{(N, \infty) \cap I_{\varepsilon}}\left|M_{\mu}^{+} f\right|^{p} u d \mu \leq 2^{p} \sum_{k} \int_{E_{k} \cap I_{\varepsilon}} 2^{k p} u d \mu=2^{p} \sum_{k, j} \int_{F_{j, k}} 2^{k p} u d \mu \\
\leq 2^{p} \sum_{k, j} \int_{F_{j, k}} u(x)\left[\frac{\int_{\left[x, b_{j, k}\right\}} f d \mu}{\int_{\left[x, b_{j, k}\right\}} \sigma d \mu}\right]^{p}\left[\frac{\int_{\left[x, b_{j, k}\right\}} \sigma d \mu}{\mu\left[x, b_{j, k}\right\}}\right]^{p} d \mu(x) .
\end{gathered}
$$

Let the measure $\nu$ be defined by

$$
d \nu(j, k, x)=\left[\frac{\int_{\left[x, b_{j, k}\right\}} \sigma d \mu}{\mu\left[x, b_{j, k}\right\}}\right]^{p} \chi_{F_{j, k}}(x) u(x) d \mu(x)
$$

on $\mathbf{Z} \times \mathbf{Z} \times \mathbf{R}$, and let $T$ denote the linear operator given by

$$
T g(j, k, x)=\frac{\int_{\left[x, b_{j, k}\right\}} g \sigma d \mu}{\int_{\left[x, b_{j, k}\right\}} \sigma d \mu} .
$$

$T$ is clearly bounded from $L^{\infty}(\sigma d \mu)$ to $L^{\infty}(d \nu)$. We shall show that $T$ is bounded from $L^{1}(\sigma d \mu)$ to weak- $L^{1}(d \nu)$. Then $T$ is bounded from $L^{p}(\sigma d \mu)$ to $L^{p}(d \nu)$, so $(4.3)$ shows

$$
\begin{aligned}
\int_{(N, \infty) \cap I_{\varepsilon}}\left|M_{\mu}^{+} f\right|^{p} u d \mu & \leq 2^{p} \int\left|T\left(f \sigma^{-1}\right)\right|^{p} d \nu \\
& \leq C \int\left|f \sigma^{-1}\right|^{p} \sigma d \mu=C \int f^{p} v d \mu .
\end{aligned}
$$

Thus, first letting $\varepsilon \rightarrow 0$, then $N \rightarrow-\infty,(1.2)$ is obtained.

It remains to prove the weak type inequality

$$
\int_{\{(j, k, x):|T g|>\lambda\}} d \nu \leq C \lambda^{-1} \int g \sigma d \mu \quad \forall \lambda>0 .
$$

Let $A_{j, k}=F_{j, k} \cap\{x:|T g(j, k, x)|>\lambda\}$. Since the $F_{j, k}$ are pairwise disjoint, so are the $A_{j, k}$. Set $d_{j, k}=\inf A_{j, k}$ and

$$
J_{j, k}= \begin{cases}{\left[d_{j, k}, b_{j, k}\right\},} & \text { if } d_{j, k} \in A_{j, k}, \\ \left(d_{j, k}, b_{j, k}\right\}, & \text { if } d_{j, k} \notin A_{j, k} .\end{cases}
$$

The intervals $J_{j, k}$ and $J_{l, m}$ are either disjoint or one contains the other. To see this, suppose $J_{j, k} \cap J_{l, m} \neq \varnothing$. Then, since $J_{j, k} \subset I_{j, k}$, we must have $I_{j, k} \cap I_{l, m} \neq \varnothing$, so one of these contains the other, say $I_{l, m} \subset I_{j, k}$. But then since $F_{j, k} \cap I_{l, m}=\varnothing$, it follows that $J_{l, m} \subset J_{j, k}$. 
Let $\mathscr{J}=\left\{J_{j, k}\right\}_{j, k}$. Select $\widetilde{J}_{1} \in \mathscr{J}$ with $\left|\widetilde{J}_{1}\right|>\frac{1}{2} \sup \{|J|: J \in \mathscr{J}\}$ and set $J_{1}=\bigcup\left\{J \in \mathscr{J}: \widetilde{J}_{1} \subseteq J\right\}$. If $J_{1}, \ldots, J_{n}$ have been formed, set $\mathscr{J}_{n}=\mathscr{J} \backslash\left(\bigcup_{j=1}^{n} J_{j}\right)$, and select, if possible, $\widetilde{J}_{n+1} \in \mathscr{J}_{n}$ with $\left|\widetilde{J}_{n+1}\right|>\frac{1}{2} \sup \{|J|$ : $\left.J \in \mathscr{J}_{n}\right\}$, and set $J_{n+1}=\bigcup\left\{J \in \mathscr{J}_{n}: \widetilde{J}_{n+1} \subseteq J\right\}$. This procedure produces a (possibly finite) sequence $\left\{J_{n}\right\}$ of pairwise disjoint intervals with $\mathscr{J}=\bigcup_{n} J_{n}$. Since $\left|J_{n}\right|<\infty$ and has left endpoint in $I_{\varepsilon}$, the assertion following (4.1) shows that

$$
\int_{J_{n}}\left|M_{\mu}^{+} \chi_{J_{n}} \sigma\right|^{p} u d \mu \leq K \int_{J_{n}} \sigma d \mu
$$

Now, if $x \in A_{j, k}$,

$$
\int_{\left[x, b_{j, k}\right\}} g \sigma d \mu>\lambda \int_{\left[x, b_{j, k}\right\}} \sigma d \mu,
$$

so the Monotone Convergence Theorem shows

$$
\int_{J_{j, k}} g \sigma d \mu \geq \lambda \int_{J_{j, k}} \sigma d \mu
$$

and hence also

$$
\int_{J_{n}} g \sigma d \mu \geq \lambda \int_{J_{n}} \sigma d \mu
$$

since $J_{n}$ may be expressed as a limit of an increasing sequence of such $J_{j, k}$.

Thus,

$$
\begin{aligned}
& \int_{\{(j, k, x):|T g|>\lambda\}} d \nu=\sum_{j, k} \int_{A_{j, k}}\left[\frac{\int_{\left[x, b_{j, k}\right\}} \sigma d \mu}{\mu\left[x, b_{j, k}\right\}}\right]^{p} u(x) d \mu(x) \\
& =\sum_{n} \sum_{\left\{(j, k): J_{j, k} \subseteq J_{n}\right\}} \int_{A_{j, k}}\left[\frac{\int_{\left[x, b_{j, k}\right\}} \chi_{J_{n}} \sigma d \mu}{\mu\left[x, b_{j, k}\right\}}\right]^{p} u(x) d \mu(x) \\
& \leq \sum_{n} \int_{J_{n}}\left|M_{\mu}^{+}\left(\chi_{J_{n}} \sigma\right)\right|^{p} u d \mu
\end{aligned}
$$

since the $A_{j, k}$ are disjoint; by (4.5) and (4.6) this does not exceed

$$
K \sum_{n} \int_{J_{n}} \sigma d \mu \leq \frac{K}{\lambda} \sum_{n} \int_{J_{n}} g \sigma d \mu \leq \frac{K}{\lambda} \int g \sigma d \mu .
$$

This proves (4.4), and completes the proof of part (a).

The proof of part (b) and the necessity of (1.8) in part (c) are similar to the corresponding proofs in part (a) and are therefore omitted. If $(u, v) \in S_{\mu}$, simple convergence arguments show that

$$
\int_{J}\left|M_{\mu}\left(\chi_{J} \sigma\right)\right|^{p} u d \mu \leq K \int_{J} \sigma d \mu<\infty
$$


for all intervals $J$ of finite length. Hence $(u, v) \in S_{p}^{+}(\mu) \cap S_{p}^{-}(\mu)$, so (1.2) for $M_{\mu}$ follows from parts (a) and (b) since $M_{\mu} f \leq M_{\mu}^{+} f+M_{\mu}^{-} f$.

\section{Proof of Theorem 3}

If $(u, v) \in S_{p}^{+}(\mu)$, then part (a) of Theorems 1 and 2 show that $(u, v) \in$ $A_{p}^{+}(\mu)$.

Now suppose $u=v, \sigma=u^{-1 /(p-1)}$, and $(u, u) \in A_{p}^{+}(\mu)$. A simple convergence argument then shows that

$$
\left(\int_{[a, b]} u d \mu\right)^{1 / p}\left(\int_{[b, c]} \sigma d \mu\right)^{1 / p^{\prime}} \leq K \mu[a, c]
$$

for all $-\infty<a \leq b \leq c<\infty$.

To prove that $(u, u) \in S_{p}^{+}(\mu)$, fix $a$ with $\int_{(-\infty, a]} u d \mu>0$. Then (5.1) shows

$$
\int_{[a, c]} \sigma d \mu<\infty \quad \forall c \geq a .
$$

Hence it suffices to show there is a constant $K_{1}$ such that

$$
\int_{[a, b]}\left|M_{\mu}^{+} \chi_{[a, b]} \sigma\right|^{p} u d \mu \leq K_{1} \int_{[a, b]} \sigma d \mu
$$

for all $b>a$, for then the Monotone Convergence Theorem gives the same inequality for intervals of the form $[a, b)$ in place of $[a, b]$. Moreover, it suffices to prove (5.3) for $b$ with $\int_{[b, \infty)} \sigma d \mu>0$. To see this, suppose (5.3) holds for all such $b$ and suppose $c>a$ with $\int_{[c, \infty)} \sigma d \mu=0$. Let $b_{0}=\inf \{x$ : $\left.\int_{[x, \infty)} \sigma d \mu=0\right\}$. If $b_{0}<a, \sigma=0 \mu$-almost everywhere on $[a, \infty)$ so there is nothing to prove, so assume $a \leq b_{0} \leq c$. If $\int_{\left[b_{0}, \infty\right)} \sigma d \mu>0$, then (5.3) holds for $b=b_{0}$ by hypothesis and since $\sigma=0 \mu$-almost everywhere on $\left(b_{0}, \infty\right)$, (5.3) for $b=b_{0}$ and for $b=c$ coincide. If $\int_{\left[b_{0}, \infty\right)} \sigma d \mu=0$, then (5.3) holds for all $b<b_{0}$ by hypothesis and the Monotone Convergence Theorem yields

$$
\int_{\left[a, b_{0}\right)}\left|M_{\mu}^{+} \chi_{\left[a, b_{0}\right)} \sigma\right|^{p} u d \mu \leq K_{1} \int_{\left[a, b_{0}\right)} \sigma d \mu
$$

which is equivalent to (5.3) with $b=c$ since $\sigma=0 \mu$-almost everywhere on $\left[b_{0}, \infty\right)$ in this case. Thus in any case (5.3) holds for $b=c$.

Suppose then that $\int_{[b, \infty)} \sigma d \mu>0$. Then (5.1) shows

$$
\int_{[a, b]} u d \mu<\infty
$$

We will show that

$$
\left(M_{\mu}^{+} \chi_{[a, b]} \sigma\right)(x) \leq C K^{p^{\prime}}\left[\left(M_{\nu} \chi_{[a, b]} u^{-1}\right)(x)\right]^{p^{\prime} / p} \quad \forall x \in[a, b \mathrm{j},
$$

where $d \nu=\chi_{[a, b]} u d \mu$ and $K$ is given by (5.1). Since (5.4) shows $\nu$ is finite, $M_{\nu}$ is bounded on $L^{1}(d \nu)$ into weak- $L^{1}(d \nu)$ and from $L^{\infty}(d \nu)$ to $L^{\infty}(d \nu)$ 
and hence also from $L^{p^{\prime}}(d \nu)$ into $L^{p^{\prime}}(d \nu)$ with norm depending only on $p$ $([9,2])$. Thus $(5.5)$ yields

$$
\begin{aligned}
\int_{[a, b]}\left|M_{\mu}^{+} \chi_{[a, b]} \sigma\right|^{p} u d \mu & \leq C K^{p p^{\prime}} \int\left|M_{\nu} \chi_{[a, b]} u^{-1}\right|^{p^{\prime}} d \nu \\
& \leq C K^{p p^{\prime}} \int_{[a, b]} u^{1-p^{\prime}} d \mu .
\end{aligned}
$$

Since $u^{1-p^{\prime}}=\sigma,(5.3)$ is obtained.

It remains to show (5.5). Since (5.2) shows $\chi_{[a, b]} \sigma \in L^{1}(d \mu)$, we have $\left(M_{\mu}^{+} \chi_{[a, b]} \sigma\right)(x)<\infty \quad \mu$-almost everywhere. Let $x \in[a, b]$ with

$$
\lambda \equiv\left(M_{\mu}^{+} \chi_{[a, b]} \sigma\right)(x)>0 .
$$

Then there is $c, x \leq c \leq b$, with

$$
\int_{[x, c]} \sigma d \mu>\frac{3}{4} \lambda \mu[x, c]>0 .
$$

Let $S=\left\{y \in[x, c]: \mu[x, y] \geq \frac{1}{2} \mu[x, c]\right\}$ and $c_{0}=\inf S$. Then

$$
\mu\left[x, c_{0}\right] \geq \frac{1}{2} \mu[x, c]>0 .
$$

If $c_{0}>x$, then $\mu\left[x, c_{0}\right) \leq \frac{1}{2} \mu[x, c]$ and we have

$$
\begin{aligned}
\int_{\left[c_{0}, c\right]} \sigma d \mu & =\int_{[x, c]} \sigma d \mu-\int_{\left[x, c_{0}\right)} \sigma d \mu \\
& >\lambda\left(\frac{3}{4} \mu[x, c]-\mu\left[x, c_{0}\right)\right) \geq \frac{1}{4} \lambda \mu[x, c] .
\end{aligned}
$$

Since $\mu\left[x, c_{0}\right]>0$ and $\int_{\left[x, c_{0}\right]} \sigma d \mu<\infty$, we have $\sigma<\infty \mu$-almost everywhere on $\left[x, c_{0}\right]$ and hence $u>0 \mu$-almost everywhere on $\left[x, c_{0}\right]$. Thus $\int_{\left[x, c_{0}\right]} u d \mu>0$, so (5.1) applied to (5.8), or to (5.6) in case $c_{0}=x$, yields

$$
\begin{aligned}
\left(M_{\mu}^{+} \chi_{[a, b]} \sigma\right)(x) & \leq 4 \frac{\int_{\left[c_{0}, c\right]} \sigma d \mu}{\mu[x, c]} \leq 4 K^{p^{\prime}}\left(\frac{\mu[x, c]}{\int_{\left[x, c_{0}\right]} u d \mu}\right)^{p^{\prime} / p} \\
& \leq 2^{p^{\prime}+1} K^{p^{\prime}}\left(\frac{\mu\left[x, c_{0}\right]}{\int_{\left[x, c_{0}\right]} u d \mu}\right)^{p^{\prime} / p}
\end{aligned}
$$

by (5.7), so (5.5) follows. This completes the proof of part (a).

The proofs of parts (b) and (c) are similar so are omitted.

\section{REFERENCES}

1. K. F. Andersen and B. Muckenhoupt, Weighted weak type Hardy inequalities with applications to Hilbert transforms and maximal functions, Studia Math. 72 (1982), 9-26.

2. A. Bernal, A note on the one-dimensional maximal function, Proc. Roy. Soc. Edinburgh Sect. A 111 (1989), 325-328.

3. H.-M. Chung, R. A. Hunt, and D. S. Kurtz, The Hardy Littlewood maximal function on $L(p, q)$ spaces with weights, Indiana Univ. Math. J. 31 (1982), 109-120. 
4. F. J. Martín-Reyes, P. Ortega Salvador, and A. de la Torre, Weighted inequalities for one-sided maximal functions, Trans. Amer. Math. Soc. 319 (1990), 517-534.

5. B. Muckenhoupt, Weighted norm inequalities for the Hardy maximal function, Trans. Amer. Math. Soc. 165 (1972), 207-226.

6. E. Sawyer, A characterization of a two weight norm inequality for maximal operators, Studia Math. 75 (1982), 1-11.

7. __, Weighted Lebesgue and Lorentz norm inequalities for the Hardy operator, Trans. Amer. Math. Soc. 281 (1984), 329-337.

8. __ Weighted inequalities for the one-sided Hardy-Littlewood maximal functions, Trans. Amer. Math. Soc. 297 (1986), 53-61.

9. P. Sjögren, $A$ remark on the maximal function for measures in $R^{n}$, Amer. J. Math. 105 (1983), 1231-1233.

10. R. Wheeden and A. Zygmund, Measure and integral, Monographs and Textbooks in Pure and Appl. Math., no. 43, Marcel Dekker, New York, 1977.

Department of Mathematics, University of Alberta, Edmonton, Alberta, Canada T6G-2G1 